\title{
Conformal sparse metasurfaces for wavefront manipulation
}

\author{
Vladislav Popov ${ }^{1}$, Shah Nawaz Burokur ${ }^{2}$. and Fabrice Boust ${ }^{1,3}$ \\ ${ }^{1}$ SONDRA, CentraleSupélec, Université Paris-Saclay, F-91190, Gif-sur-Yvette, France \\ ${ }^{2}$ LEME, UPL, Univ Paris Nanterre, F92410 Ville d'Avray, France and \\ ${ }^{3}$ DEMR, ONERA, Université Paris-Saclay, F-91123, Palaiseau, France
}

\begin{abstract}
The last decade was marked by the advent of the concepts of phase gradient and Huygens' metasurfaces which simple theoretical models have been leading the research on metasurfaces ever since. Meanwhile, theoretical modelling of non-planar metasurfaces have appeared to be exceptionally challenging where it demands accurate analysis of the metasurface geometry. The present work addresses this challenge with a radically different theoretical approach demonstrating how numerical calculation of Green's function can be employed to design conformal metasurfaces of arbitrary geometries within the same framework and without any accommodation. Against the classical concepts, the new approach permits building a metasurface of meta-atoms with electric-only response and significantly simplifying its design and fabrication. The theory is endorsed experimentally by testing several metasurface prototypes for different beam-forming functionalities: radiation with single or multiple beams in any desired directions.
\end{abstract}

\section{INTRODUCTION}

In the last two decades, metasurfaces that are thin two-dimensional metamaterials have proven themselves as a powerful tool to tailor wavefronts $\frac{12}{12}$. A myriad of metasurface-based planar devices has been proposed and validated. Nowadays there is an increasing interest in conformal metasurfaces to perfectly match curved shapes ${ }^{3}$ 11. As expected, the design and fabrication of conformal metasurfaces are more demanding compared to planar metasurfaces ${ }^{6}$. Adopting flexible substrates to implement metasurfaces opens a new way for integration with other elements and non-planar designs including lightweight wearable devices $\frac{61810}{}$. In addition, for example, microwave antennas based on conformal metasurfaces do not only allow one to meet the aerodynamic specifications of aircrafts and satellites ${ }^{3 / 11}$ but also break the fundamental constraint of their planar counterparts. For instance, the aperture of a flat metasurface antenna vanishes when the beam steering angle increases ${ }^{12}$.

The design of a conformal metasurface is generally based on geometrical optics approach 3 -111, where a proper spatial distribution of local reflection (transmission) coefficient should be established along the reflecting (transmitting) metasurface. It has been revealed that this heuristic approach has strong limitations in terms of efficiency and versatility 13114. On the other hand, more rigorously, metasurfaces can be described by means of continuous surface impedances ${ }^{15 \mid 16}$. Unfortunately, theoretical modelling of conformal metasurfaces appears to be exceptionally challenging, demanding accurate analysis of the metasurface geometry and dealing with curvilinear coordinates ${ }^{10}$.

The contribution of this work is twofold. First, it is shown how numerical calculation of a Green's function can be employed to design conformal sparse metasurfaces capable of creating arbitrary field patterns for arbitrary external excitations. The proposed approach does not use any complex local coordinate system matched to a particular geometry such that arbitrarily-shaped metasurfaces can be considered without any accommodation. Sparse metasurfaces possess strongly non-local response ${ }^{17 \mid}[23$ and can be described in terms of neither surface impedance nor local reflection and/or transmission coefficients being not subject to fundamental efficiency limitations as their "dense" counterparts13|14|21/24|25. At the same time, the sparseness allows to establish a global theoretical model and get a microscopic insight into the theoretical analysis of conformal metasurfaces. Secondly, it is detailed how to realize these conformal sparse metasurfaces. To describe the design procedure, three semicylindrical sparse metasurfaces illuminated by an arbitrary complex wave configuration are experimentally demonstrated at microwave frequencies.

\section{THEORY}

Without loss of generality, we consider the case of TE polarization and a two-dimensional (2D) geometry. A translation symmetry is assumed along one of the three spatial dimensions. We compose a sparse metasurface by a finite set of $N$ loaded wires distributed along the surface of an arbitrarily-shaped dielectric substrate. The wires are oriented along the translation-invariant direction. An example presented in Fig. 11 illustrates an array of loaded wires distributed along an arbitrarily-shaped substrate. Microscopically, a loaded wire represents itself as a chain of subwavelength meta-atoms. On the other hand, macroscopically, we model the loaded wire as uniform and having a deeply subwavelength effective radius $r_{\text {eff }}$.

We consider external sources radiating a background wave with the electric field directed along $x$-axis and exciting polarization currents in the loaded wires. However, here we do not impose any condition on the external sources unlike plane-wave excitation used for majority of metasurfaces presented in literature. The polarization currents excited in the wires can shape the field radiated by a metasurface. The total electric field $E_{x}(\mathbf{r})$ can 


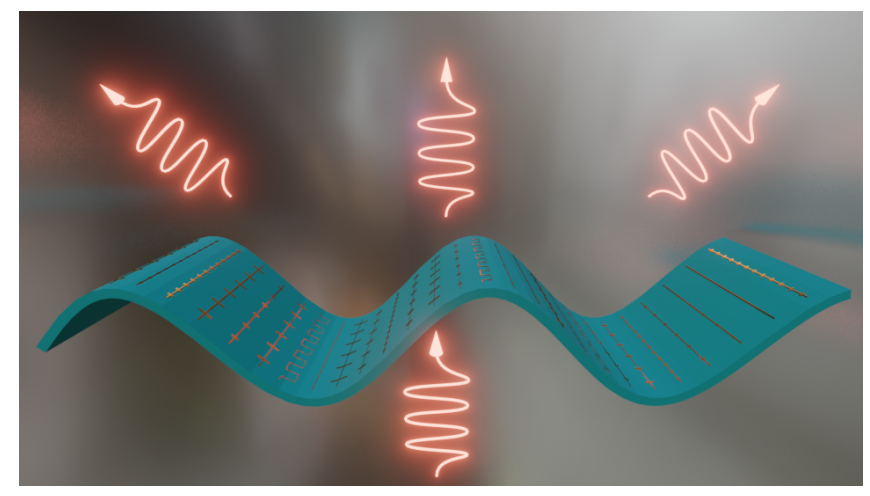

FIG. 1. Illustration of an arbitrarily-shaped sparse metasurface transforming an arbitrary impinging wave into multiple beams.

be split into two terms: the field $E_{x}^{(e x t)}(\mathbf{r})$ due to external sources alone (in the absence of wires) and the field $E_{x}^{(s c t)}(\mathbf{r})$ re-radiated (scattered) by the polarization currents. The latter is found by means of a Green's function $G_{x x}\left(\mathbf{r}, \mathbf{r}^{\prime}\right)$ of a system corresponding to a metasurfacebased device, which means that this Green's function is not the one of free space. It is calculated in the presence of a curved substrate (backed with a metal sheet in the case of a reflecting configuration) and other dielectric and/or metallic parts of the system. In the equation form, the Green's function is defined as

$$
\left(\frac{\partial^{2}}{\partial y^{2}}+\frac{\partial^{2}}{\partial z^{2}}+k_{0}^{2} \varepsilon_{r}(\mathbf{r})\right) G_{x x}\left(\mathbf{r}, \mathbf{r}^{\prime}\right)=i \omega \mu_{0} \delta\left(\mathbf{r}-\mathbf{r}^{\prime}\right)
$$

where $\mathbf{r}$ and $\mathbf{r}^{\prime}$ are $2 \mathrm{D}$ vectors in $y 0 z$ plane, $\mu_{0}$ is the permeability of free space, $k_{0}$ is the wavenumber of free space, $\omega$ is the angular frequency, $\varepsilon_{r}(\mathbf{r})$ is the relative permittivity corresponding to dielectric and metallic parts of the metasurface-based device in the absence of the loaded wires. As the considered system does not have a translational symmetry in the 2D plane, a Green's function $G\left(\mathbf{r}, \mathbf{r}_{q}\right)$ is a function of $\mathbf{r}$ and $\mathbf{r}^{\prime}$ (not their difference). Then, the scattered electric field can be represented as the convolution and since the wires are distributed only along the top face of an arbitrarily-shaped substrate, the integration should be performed only over this face

$$
E_{x}^{(s c t)}(\mathbf{r})=\int G_{x x}\left(\mathbf{r}, \mathbf{r}^{\prime}\right) J_{x}\left(\mathbf{r}^{\prime}\right) \mathrm{d} \mathbf{r}^{\prime},
$$

where $J_{x}(\mathbf{r})$ is the electric current density corresponding to polarization currents in all wires. Following the assumption of infinitesimally thin wires, we approximate the current density as $J_{x}(\mathbf{r})=\sum_{q=1}^{N} I_{q} \delta\left(\mathbf{r}-\mathbf{r}_{q}\right)$, where $I_{q}$ and $\mathbf{r}_{q}$ is the polarization line current excited in the $q^{\text {th }}$ wire and its position, respectively. Taking this into account, the total electric field can be written as

$$
E_{x}(r, \phi)=E_{x}^{(e x t)}(r, \phi)+\sum_{q=1}^{N} G_{x x}\left(r, \phi ; \mathbf{r}_{q}\right) I_{q}
$$

where $r$ and $\phi$ are polar coordinates: radius and polar angle, respectively. The total field $E_{x}(r, \phi)$ is represented by the superposition of the wave radiated by external sources $E_{x}^{(e x t)}(r, \phi)$ and waves $G\left(r, \phi ; \mathbf{r}_{q}\right) I_{q}$ scattered by the wires.

Sparse configuration of the metasurface allows one to accurately take into account the interactions between the wires via Ohm's law

$$
Z_{q} I_{q}=E_{x}^{(e x t)}\left(\mathbf{r}_{q}\right)-\sum_{p=1}^{N} Z_{q p}^{(m)} I_{p}
$$

A load-impedance density (or impedance per unit length of a wire) $Z_{q}$ is a characteristic of a loaded wire and can be engineered for instance by tuning the geometrical parameters of meta-atoms constituting a wire. The righthand side of Eq. (4) represents the total electric field at the position of the $q^{\text {th }}$ wire, where

$$
Z_{q p}^{(m)}=-G_{x x}\left(\mathbf{r}_{q}, \mathbf{r}_{p}\right)
$$

is the mutual-impedance density (the electric field created by the $p^{\text {th }}$ wire at the position of the $q^{\text {th }}$ wire). The separation between two neighboring wires can be arbitrary, as long as polarization currents in the wires can be approximated by a $2 \mathrm{D}$ delta function, which limits the transverse size of a wire. On the other hand, the interaction between neighboring wires due to the macroscopic field $G_{x x}\left(\mathbf{r}_{q}, \mathbf{r}_{p}\right)$ should be much stronger than the coupling via a strongly localized microscopic near-field. The latter appears when geometric parts constituting two neighboring wires come very close to each other. As it is demonstrated further, the simple model of $\delta$ function works surprisingly well even for complex designs. The self-action of the $q^{\text {th }}$ wire and its interaction with the environment is accounted via

$$
Z_{q q}^{(m)}=-\frac{1}{2 \pi r_{e f f}} \oint G_{x x}\left(\mathbf{r}, \mathbf{r}_{q}\right) \mathrm{d} \mathbf{r},
$$

where the integration is performed over the circumference of the wire of effective radius $r_{\text {eff }}$. Although being very simple, Eq. (4) has an important practical implication: it allows one to know in advance the impact of one polarization current on another and to accordingly adjust the load-impedance densities. Conceptually, it means that the developed approach is global, being in strong contrast with conventional theoretical models of metasurfaces which are essentially local. Interaction between wires described via the matrix of mutual-impedance densities indicates that sparse metasurfaces are intrinsically strongly non-local, i.e. the current in the $q^{\text {th }}$ wire depends on the one in the $p^{\text {th }}$ wire.

Meanwhile, by appropriately choosing $Z_{q}$ and the number of wires $N$, we are then able to tailor the field at will. Each term on the right-hand side of Eq. (3) at a given distance $r\left(r \neq\left|\mathbf{r}_{q}\right|\right.$ for all $\left.q\right)$ can be approximated by a partial Fourier sum over the polar angle $\phi: E_{x}^{(e x t)}(r, \phi)=\sum_{n=-M}^{M} C_{n}^{(e x t)} e^{i n \phi}$ and $G_{x x}\left(r, \phi ; \mathbf{r}_{q}\right)=$ 
(a)

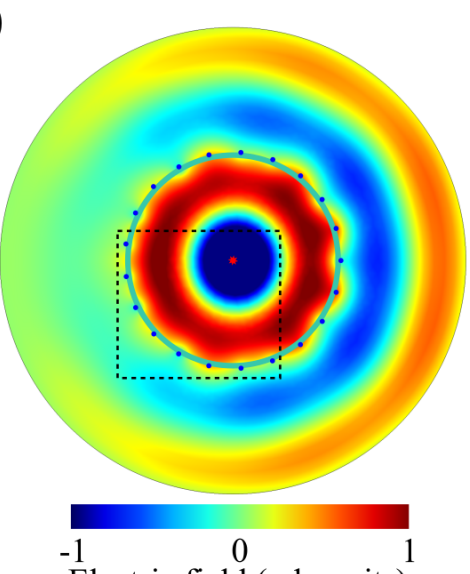

Electric field (arb. units)

(b)

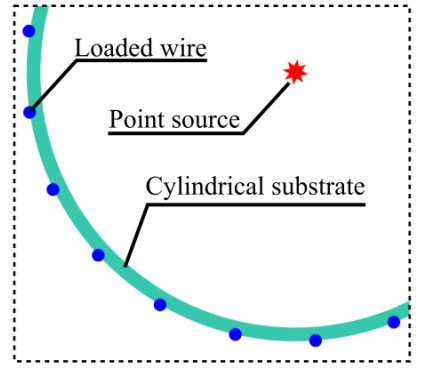

(c) 1 .

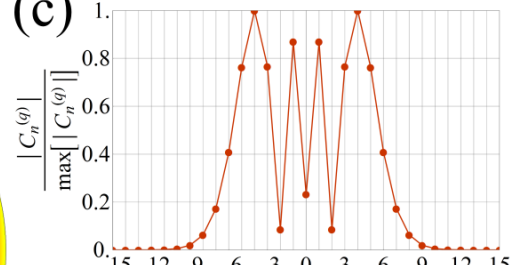

(d)

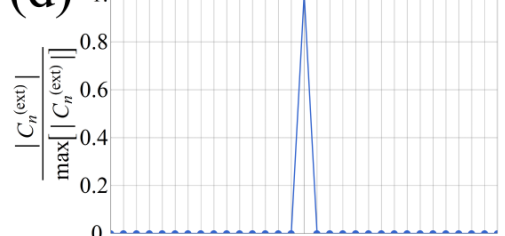

(e)

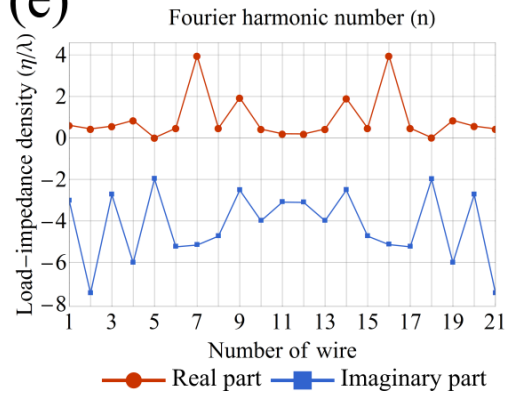

(f)

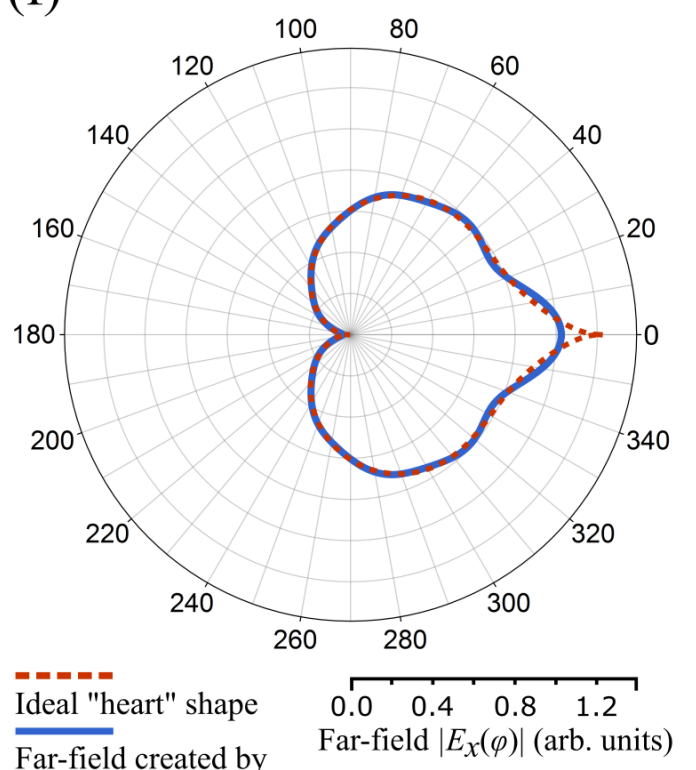

sparse metasurface

FIG. 2. (a) Profile of the electric field created by a cylindrical sparse metasurface. The metasurface is represented by 21 loaded wires uniformly distributed along a cylindrical substrate of thickness $\lambda_{0} / 120$ and having the relative permittivity 2.2. The metasurface is excited by a point source placed in its center. (b) Zoom of a part of the sparse metasurface. (c) $\left|C_{n}^{(q)}\right| / \max \left[\left|C_{n}^{(q)}\right|\right](\mathrm{d})\left|C_{n}^{(e x t)}\right| / \max \left[\left|C_{n}^{(e x t)}\right|\right]$ vs. the number $n$ of Fourier harmonic. (e) Load-impedance densities required to approximate the "heart" shaped far-field pattern. (f) Comparison of the far-field pattern created by the sparse metasurface (solid curve) and the ideal "heart" shape (dashed curve).

$\sum_{n=-M}^{M} C_{n}^{(q)} e^{i n \phi} . M$ is the maximum Fourier harmonic defined as the minimum number $M$ such that

$$
\left|C_{ \pm|M+r|}^{(e x t, q)}\right| / \max \left|C_{n}^{(e x t, q)}\right| \ll 1
$$

for all $r=1,2, \ldots$. The number $M$ might affect many parameters (such as geometrical parameters and material properties of a sample, the distance $r$ ) but the most important one is the physical aperture $\max \left[\left|\mathbf{r}_{q}-\mathbf{r}_{p}\right|\right]$. The larger the aperture the greater is $M$. Evidently, the total electric field $E_{x}(r, \phi)$ can also be represented by a partial Fourier sum $\sum_{n=-M}^{M} C_{m} e^{i n \phi}$ and the relation between the Fourier coefficients is as follows:

$$
C_{n}=C_{n}^{(e x t)}+\sum_{q=1}^{N} C_{n}^{(q)} I_{q},
$$

where $C_{n}^{(e x t)}$ and $C_{n}^{(q)}$ are known. When a sparse metasurface is composed of at least $N=2 M+1$ loaded wires one can establish arbitrary azimuthal field distributions within the functional space of the $2 M+1$ Fourier harmonics by adopting the Fourier coefficients $C_{n}$.

Corresponding load-impedance densities can be found from Eq. (4) after solving Eq. (8) with respect to $I_{q}$, which in this case has a single solution 26. As a matter of fact, there is no guarantee that analytically found $Z_{q}$ would not require implementing active and/or lossy elements since $\Re\left[Z_{q}\right] \neq 0$ in a general case. In order to additionally deal with only reactive load-impedance densities $Z_{q}=i \Im\left[Z_{q}\right]$, one might need a number of wires $N \geq 2 M+1$ for constructing arbitrary radiation patterns, as recently discussed in Ref.20. Essentially, the procedure described in this paragraph allows one to know in advance all possible configurations of the azimuthal field for a given geometry of metasurface and number of wires. It includes practical parameters such as beamwidth and sidelobes level.

To sum up, after establishing the geometry of a sparse metasurface (flat, cylindrical, or any other shape), excitation type and positions of $N$ wires, we are able to calculate a Green's function $G_{x x}\left(r, \phi ; \mathbf{r}_{q}\right)$ and the background field $E_{x}^{(e x t)}(r, \phi)$ radiated by external sources. With Eqs. (3) and (4), a relation between the radiated field and load-impedance densities is established and the procedure related to Eq. (8) is used to find the number of wires and to determine possible functional characteristics of a sparse metasurface such as beamwidth and sidelobes level in beam-forming applications. A crucial point of 


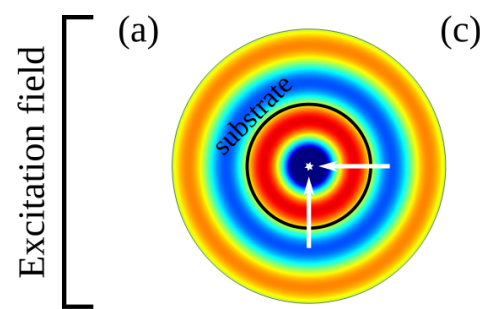

(c)

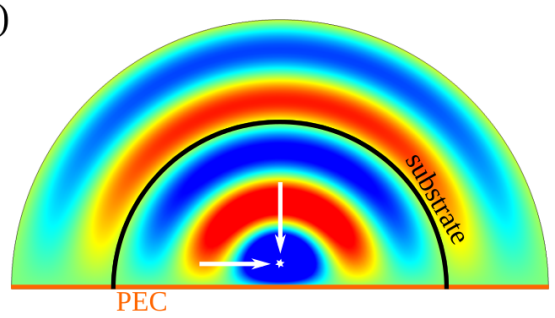

(b)

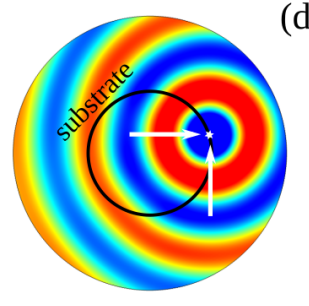

(d)

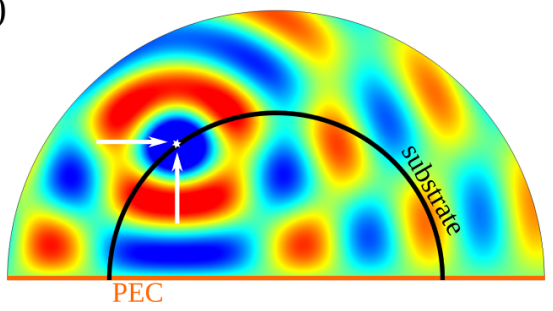

(e)

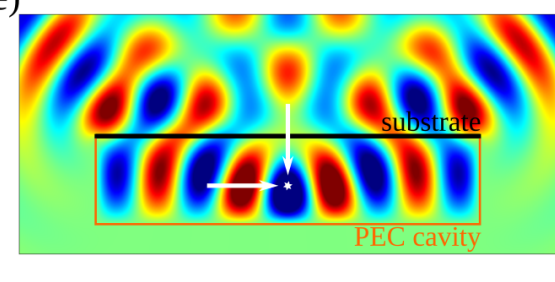

(f)

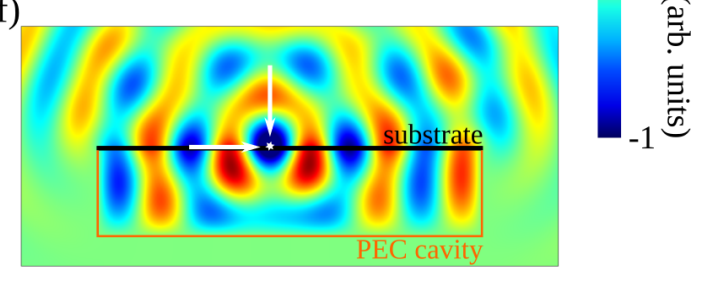

FIG. 3. Simulated distributions of the electric field created by an elementary source in different configurations: (a),(b) a cylindrical dielectric substrate in free space, (c),(d) a semi-cylindrical substrate over a PEC plane, (e), (f) an open Fabry-Perot cavity cover with a planar dielectric substrate. The panels (a),(c) and (e) demonstrate distributions of the excitation field, the external (elementary) source is shown by arrows. The panels (b),(d) and (f) demonstrate function $G_{x x}\left(\mathbf{r}, \mathbf{r}_{q}\right)$ obtained as the electric field radiated by an elementary source located at $\mathbf{r}_{q}$ (shown by arrows).

the above analysis is the Green's function which, being defined for an arbitrary finite-size system, does not have any applicable analytical form. Instead, it is suggested to compute it numerically with the help of full-wave numerical simulations as detailed in Appendix A A design procedure to practically implement loaded wires is described in Appendix B. An optimization procedure is brought to solve the inverse scattering problem assuming only reactive load-impedance densities of wires, as detailed in Appendix C.

\section{2D SIMULATION EXAMPLE}

Let us consider a simple example of a cylindrical sparse metasurface for far-field manipulation. The radius of the metasurface is fixed to $5 \lambda_{0} / 6$ ( $\lambda_{0}$ is the vacuum operating wavelength) and it is excited by a point source placed in the center, as shown in Figs. 2(a) and (b). The source creates a cylindrical background wave, as illustrated in Fig. 3(a). Due to the symmetry, Green's functions $G_{x x}\left(r, \phi ; \mathbf{r}_{q}\right)$ of different wires placed on the cylindrical substrate are simply shifted with respect to each other (as function of $\phi$ ). It means that one has to analyse the Fourier decomposition of only one Green's function and of the background wave in order to find the parameter $M$. Figure 3(b) shows an example of the function $G_{x x}\left(r, \phi ; \mathbf{r}_{q}\right)$ computed for a particular $\mathbf{r}_{q}$. Following the definition in Eq. (7), from Figs. 2(c) and (d), it can be seen that it is enough to have $N=2 \times 10+1=21$ wires in order to be able to construct all possible far-field radiation patterns within the Fourier space of 21 harmonics $\exp [i n \phi]$. As an illustrative example, one can reconstruct in the far-field region the shape of a "heart" after the re- quired corresponding load-impedance densities are found from Eqs. (8) and (4). The required load-impedance densities are plotted in Fig. 2(e) and correspond to passive elements $\left(\Re\left[Z_{q}\right]>0\right)$. The real part of $Z_{q}$ can be engineered in a similar fashion as proposed in in Ref ${ }^{27}$. The resulted far-field pattern is depicted in Fig. 2 (f) and compared to the ideal shape of a "heart". Figure 2(a) shows the corresponding profile of the electric field in the proximity to the metasurface. In order to improve the accuracy of approximating some ideal curve, one needs to increase $M$ which can be done by increasing the size of the metasurface (the radius in the considered case). Finally, as stated above, to best of our knowledge, there is no analytical formula for a Green's function of a system with a cylindrical substrate. Additional details on this example are given in Appendix A.

\section{3D SIMULATION EXAMPLES}

In this section, we provide several examples of designs of conformal sparse metasurfaces operating in the microwave frequency range, which perform different beamforming functionalities. The metasurfaces demonstrated in what follows represent a set of loaded wires uniformly distributed along the top face of a dielectric substrate. The bottom face of the substrate is metal-free. Operating in the transmission mode, the metasurfaces transform an incident wavefront from one side into a desired wavefront on the other side. The designs are developed with the optimization-aided procedure (see Appendix C), verified by means of 3D full-wave simulations and compared to predictions of the analytical model represented by Eqs. (3) and (4). The impact of using different number 
(a)

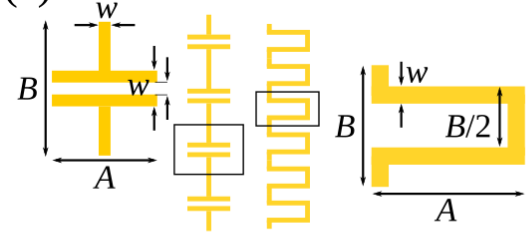

(d)

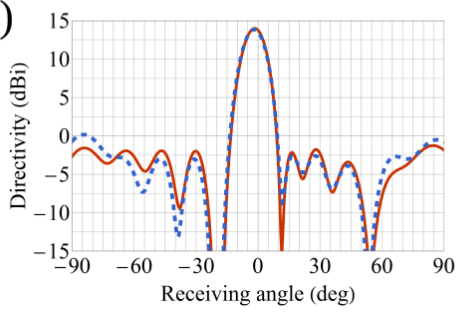

(b)

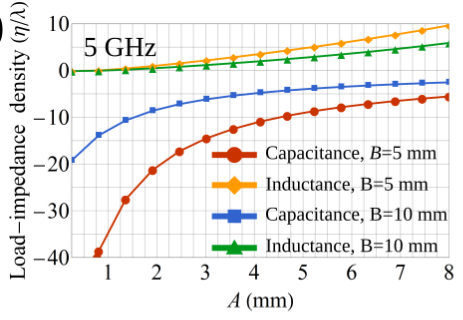

(e)

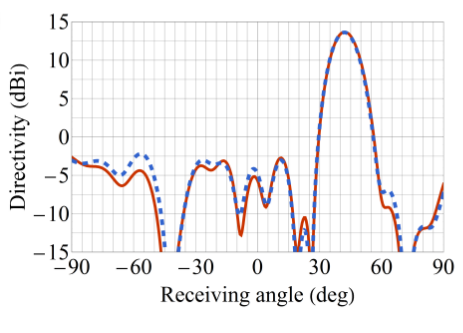

(c)

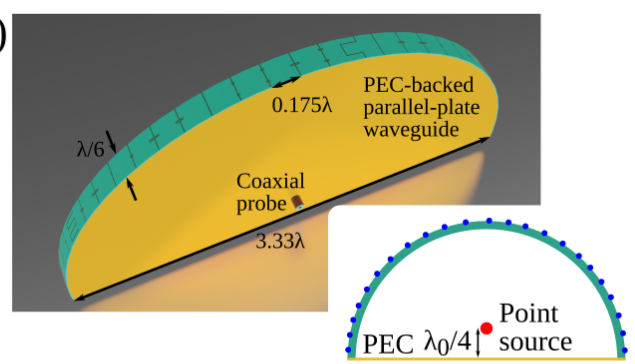

(f)

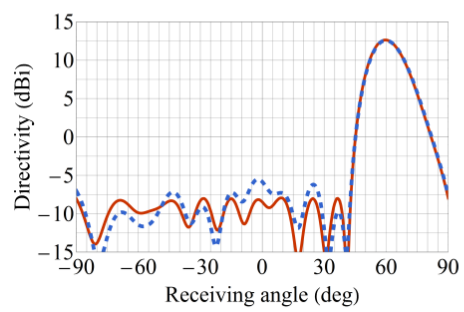

Analytical model $=$ - $=$. 3D full-wave simulation

FIG. 4. (a) Schematics of a printed capacitance (left) and inductance (right), $w=0.25 \mathrm{~mm}\left(r_{\text {eff }}=w / 4\right.$, see Ref. ${ }^{28}$ ). (b) Retrieved load-impedance densities (only imaginary parts are shown) of capacitively- and inductively-loaded wires at 5 GHz. (c) 3D schematic of a semi-cylindrical sparse metasurfaces of $100 \mathrm{~mm}$ radius and incorporating 29 wires. The inset figure shows a 2D schematic. (d)-(f) Engineered radiation patterns :two-dimensional directivity vs. angle. Predictions of the theoretical model (solid red curves) are compared to the results of 3D full-wave simulations of three different designs of sparse metasurfaces (dashed blue curves). The used substrate is F4BM220 of $0.5 \mathrm{~mm}$ thickness. Operating frequency is $5 \mathrm{GHz}, \lambda_{0} \approx 60 \mathrm{~mm}$.

of wires composing a sparse metasurface is also analysed.

The design of loaded wires is performed within the local periodic approximation, developed in Ref.29 and adopted to current examples in Appendix B. The wires are built up from printed capacitors (left design of Fig. 4(a)) and inductors (right design of Fig. 4(a)), which provide a wide range of accessible load-impedance densities as shown in Figs. 4(b) for $5 \mathrm{GHz}$ operation. A particular importance of the LPA of Ref ${ }^{29}$ should be emphasized: Sparse metasurfaces cannot be designed as their dense counterparts. Indeed, the design procedure results in the load-impedance density of a loaded wire, which is its proper characteristic and depends on neither the substrate's thickness nor the inter-wire distance, see also Ref. $[30$. On the other hand, when designing conventional dense metasurfaces, one deals with such characteristics as surface impedances (or local reflection and/or transmission coefficients) which represent the integral response a unit cell and depend on the parameters of a substrate and the inter-element distance.

\section{A. Semi-cylindrical sparse metasurface}

In the first example, we demonstrate a sparse metasurface operating at $5 \mathrm{GHz}$ (vacuum wavelength $\lambda_{0} \approx$ $60 \mathrm{~mm}$ ) and conformed to a semi-cylindrical shape of $1.67 \lambda_{0}=100 \mathrm{~mm}$ radius. $N=29$ loaded wires are uniformly distributed along the top face of a $\lambda_{0} / 120=0.5$ $\mathrm{mm}$ thick and $5.25 \lambda_{0}=315 \mathrm{~mm}$ long F4BM220 substrate, which serve to control $2 M+1=29$ independent Fourier harmonics of the far-field as follows from Eqs. (7) and (8). The metasurface is illuminated by a point source placed at $\lambda_{0} / 4$ distance above a PEC wall joining the two ends of the semi-cylinder, as shown in the inset of Fig. 4(c). Distribution of the electric field created by the source and an example of function $G_{x x}\left(\mathbf{r}, \mathbf{r}_{q}\right)$ computed for this configuration are shown in Figs. 3 (c) and (d), respectively. In order to emulate the $2 \mathrm{D}$ configuration of the considered theoretical model, a narrow strip of the metasurface (of $\lambda_{0} / 6$ width) is embedded in between two PEC plates which form a parallel-plate waveguide. The source is represented by a coaxial probe exciting TEM waveguide mode. A schematics of the system is shown in Fig. 4(c).

Figures 4(d)-(f) demonstrate three different configurations of the far-field corresponding to three different designs of semi-cylindrical sparse metasurfaces. The directivity plotted in Figs. 4(d)-(f) is the two-dimensional directivity, which is calculated as follows

$$
D(\varphi)=2 \pi\left|E_{f f}(\varphi)\right|^{2} / \int_{0}^{2 \pi}\left|E_{f f}(\varphi)\right|^{2} \mathrm{~d} \varphi,
$$

where $E_{f f}(\varphi)$ is $E_{x}(r, \varphi)$ from Eq. (3) calculated in the far-field region $(r \rightarrow \infty)$. The 3D simulation results (dashed blue curves) are compared to the corresponding far-field patterns predicted by the analytical model in Eq. (3) (solid red curves), where an almost perfect agreement is observed. It proves a high accuracy of the established design procedure based on the analytical model represented by Eqs. (3) and (4) on one hand and the LPA on the other hand.

Because of the very narrow $\lambda_{0} / 6$ aperture in the transverse direction, the radiation pattern presents a wide 

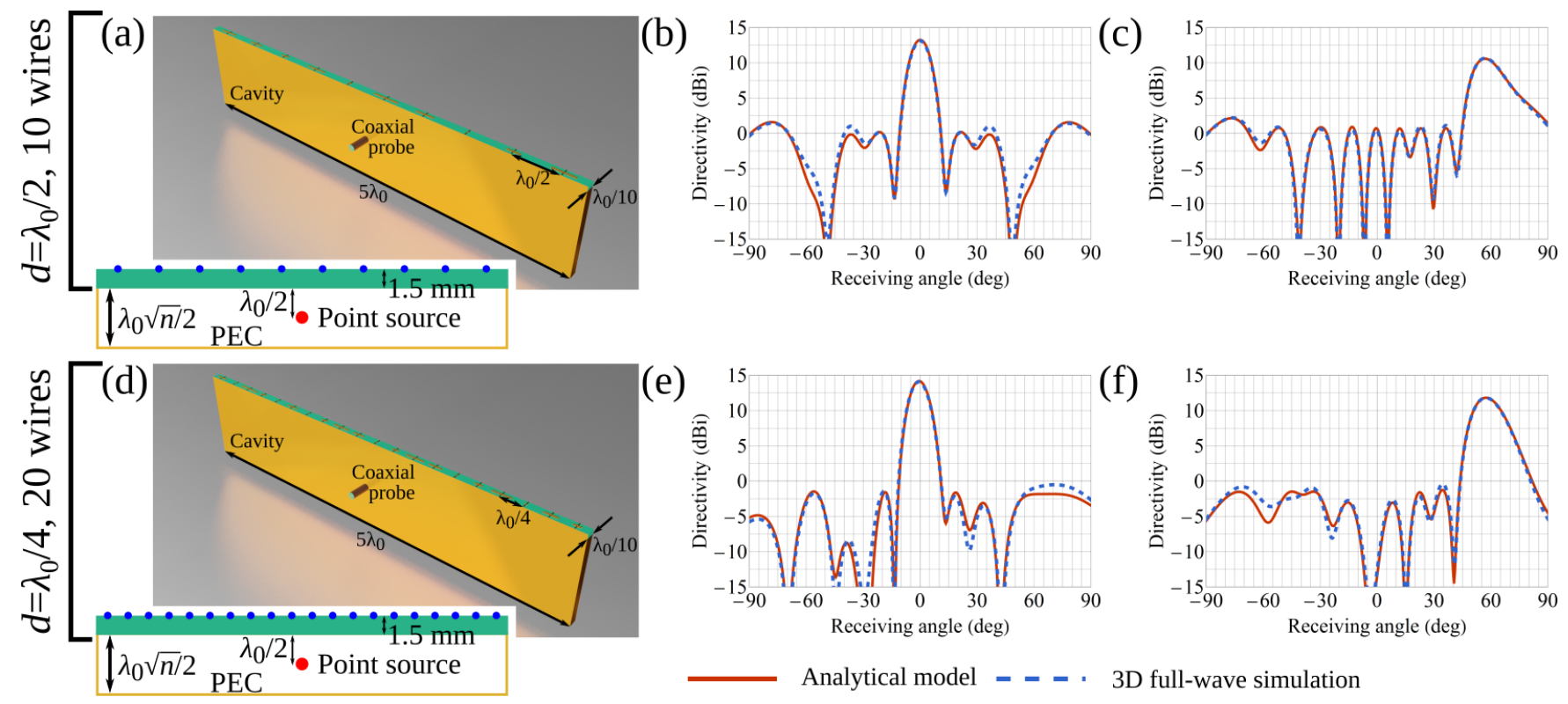

Analytical model - - - . 3D full-wave simulation

FIG. 5. (a), (d) 3D schematics of cavity-excited flat sparse metasurfaces having 10 (a) and 20 (d) wires. The inset figures show $2 \mathrm{D}$ schematics of the corresponding configurations. (b),(c),(e),(f) Two-dimensional directivity vs. angle of engineered radiation patterns corresponding to analytical and 3D simulation results of four different designs of sparse metasurfaces. Predictions of the theoretical model (solid red curves) are compared to the results of 3D full-wave simulations of three different designs of sparse metasurfaces (dashed blue curves). The panels (a)-(c) and (d)-(f) correspond, respectively, to the designs incorporating 10 wires (the inter-wire distance is $\lambda_{0} / 2$ ) and 20 wires (the inter-wire distance is $\lambda_{0} / 4$ ). The used substrate is F4BM220 of 1.5 mm thickness, the cavity height is $\lambda_{0} \sqrt{n} / 2 \approx 33.54 \mathrm{~mm}$, where $n$ is the cavity length $\left(5 \lambda_{0}\right)$ divided over the wavelength $\lambda_{0}$. Operating frequency is $10 \mathrm{GHz}, \lambda_{0} \approx 30 \mathrm{~mm}$.

beam in the E-plane to produce a 3D fan-shaped beam. However, if the distance between two PEC plates exceeds $\lambda_{0} / 2$, higher order waveguide modes can be excited, leading to a nonuniform field distribution along the $x$-direction that was assumed to be translation invariant. This problem can be overcome and a much narrower beam can be achieved in the E-plane by elaborating on the excitation source as discussed in the following Section.

\section{B. Cavity-excited sparse metasurface}

In the second example, we consider planar sparse metasurfaces placed on the top of an open rectangular PEC cavity excited by a coaxial probe. Distribution of the excitation field created by the probe inside and outside the cavity are demonstrated in Fig. 3(e). A particular example of function $G_{x x}\left(\mathbf{r}, \mathbf{r}_{q}\right)$ calculated for this configuration is presented by Fig. 3(f). Results for two configurations with 10 and 20 wires are compared. For the given aperture size of $5 \lambda_{0}$, the number $2 M+1$ of independent Fourier harmonics forming the far-field equals to 42 . While neither 10 nor 20 wires is enough to arbitrarily control 42 Fourier harmonics, it is however possible to perform efficient beam-forming. Schematics of the configurations are shown in Figs. 5(a) and (d). Figures 5(b),(c) and (e),(f) demonstrate engineered radia- tion patterns obtained by means of the developed analytical model for 10- and 20-element sparse metasurfaces, respectively. The verification is performed via 3D full-wave simulations (blue dashed curves) where an almost perfect agreement with the analytical curves (red solid) is observed. The comparison of Figs. 5(b) and (e) and Figs. 5(c) and (f) represents an important result: two times more wires lead to a maximum of only $2 \mathrm{dBi}$ improvement of the directivity. Therefore, one should carefully choose the number of wires as comparable performances can be achieved with a lesser effort, which can be particularly advantageous for reconfigurable designs. Fortunately, the presented analytical model allows one to optimize the number of elements by considering a beforehand computed Green's function and without involving time-consuming 3D full-wave simulations of real designs.

For the sake of comparison with literature, we would like to mention that cavity-excited metasurfaces have been considered in works done by A. Epstein et al. .25|32 $^{\text {. }}$ In strong contrast to the single-layer design of sparse metasurfaces presented in Fig. 5, metasurfaces are designed as dense three-layer structures to emulate magnetic and/or bianisotropic response in Refs. ${ }^{25 / 32}$. 


\section{(a) \\ (d)}
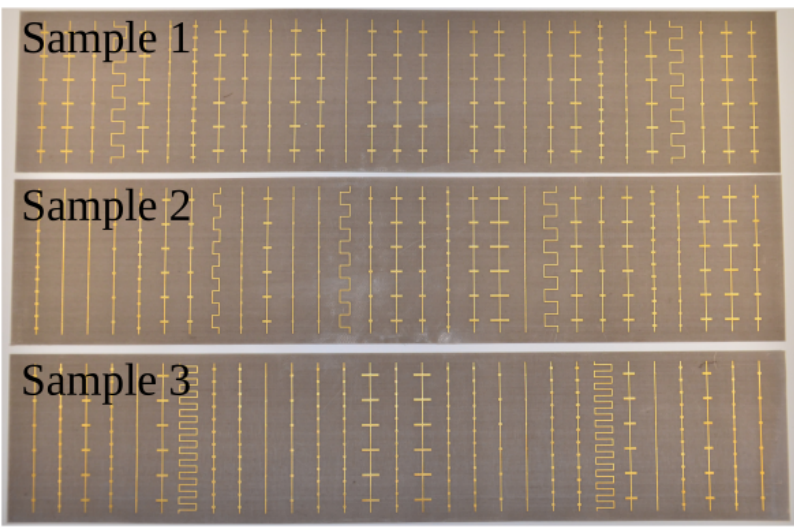

Face 1: printed dipoles

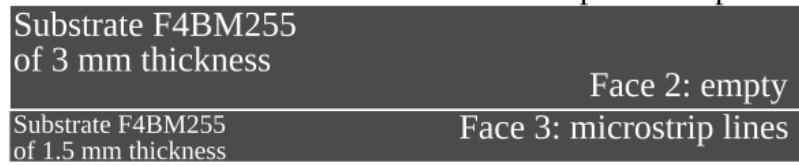

Face 4: ground plate

(e)

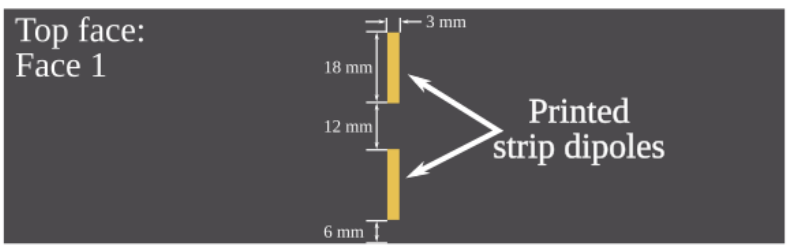

(b)

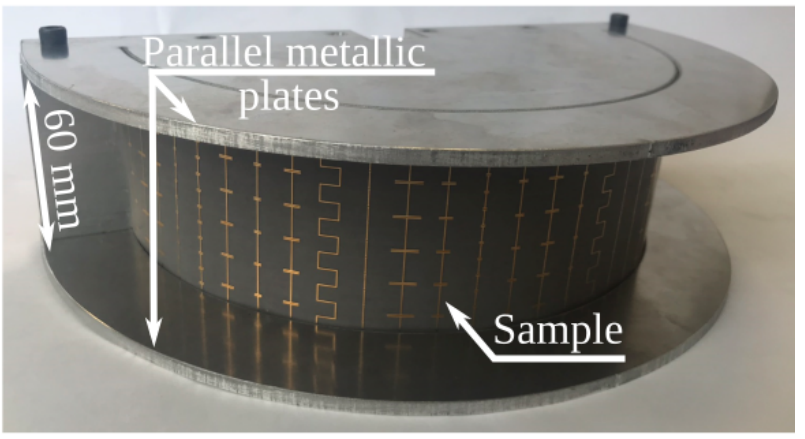

(c)

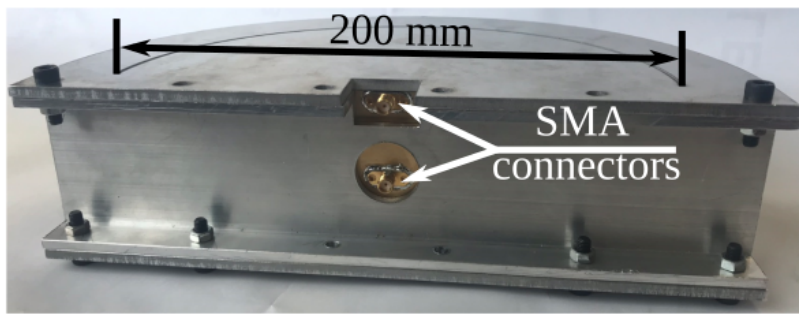

(f)

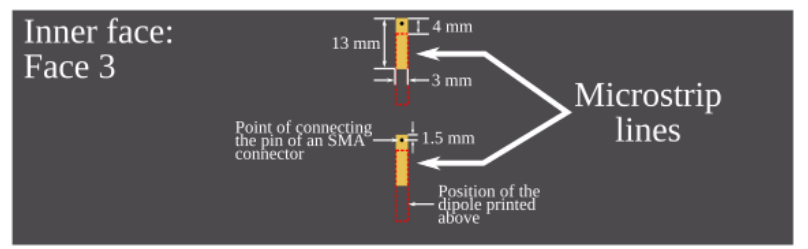

FIG. 6. (a) A photography of fabricated sparse metasurfaces on a thin flexible substrate. (b), (c) Photographies of an assembled experimental prototype: (b) the front side and (c) the back side. (d) A schematic of the side view of a PCB of the excitation source. (e), (f) Schematics of the top face (e) and the inner face (f) of the PCB. The schematics contain all the information necessary to design the source used to excite the samples in the experiment. The design is performed following Ref. 31 .

\section{EXPERIMENTAL EXAMPLES}

To validate the approach, we designed and fabricated three sparse metasurfaces using extremely thin $\left(\lambda_{0} / 240=0.25 \mathrm{~mm}\right)$ flexible substrates F4BM220, as highlighted by the photographies in Figs. 6(a) and (b). The samples operating in the microwave frequency range with a central frequency fixed at $5 \mathrm{GHz}$ were fabricated by means of conventional printed circuit board (PCB) technology. Each sample is composed of twenty nine wires equidistantly distributed along a $5.25 \lambda_{0} \approx 315 \mathrm{~mm}$ long substrate, making the separation between two neighbouring wires approximately equal to $\lambda_{0} / 5$. The wires are built up from printed capacitors and inductors (see Fig. 4(a)), which provide a wide range of accessible loadimpedance densities as shown in Fig. 4(b).

The ultra-thin samples are then conformed to create semi-cylindrical surfaces of $100 \mathrm{~mm}$ radius. A photography of the assembled prototype is presented in Figs. 6.(b) and (c). The external source exciting the samples is made of two microstrip dipole antennas printed on a metal-backed substrate 31 , whose design is detailed in Figs. 6(d)-(f). The distance between the source and sparse metasurface being $100 \mathrm{~mm}\left(\approx 1.7 \lambda_{0}\right)$, the background field pattern is neither a plane wave nor a cylindrical wave, as highlighted by Fig. 7 .

The three samples were designed to show different beam-forming examples: a single broadside beam at $0^{\circ}$, a steered beam at $40^{\circ}$ and a multibeam configuration with two beams at $\pm 30^{\circ}$ from broadside. The inverse scattering problem was solved by maximizing the power in the desired direction and minimizing the sidelobes level (with respect to the geometrical parameters $A$ and $B$ of the loaded wires) radiated by the sparse metasurface in desired directions, and the maximization procedure was implemented as particle swarm optimization 33 detailed in Appendix C. The different configurations were experimentally validated by radiation patterns measurements performed in an anechoic chamber. A horn antenna used a receiver is kept fixed and the assembled prototype is mounted on a rotating platform as shown in Fig. 7(a). Figures 7(b)-(d) present the experimental results obtained from the three samples. The level of spurious scattering (at the operating frequency) does not exceed $-12 \mathrm{~dB}$ for the first sample, $-9 \mathrm{~dB}$ for the second one and $-13 \mathrm{~dB}$ for the third one. The radiation pattern 
(a)

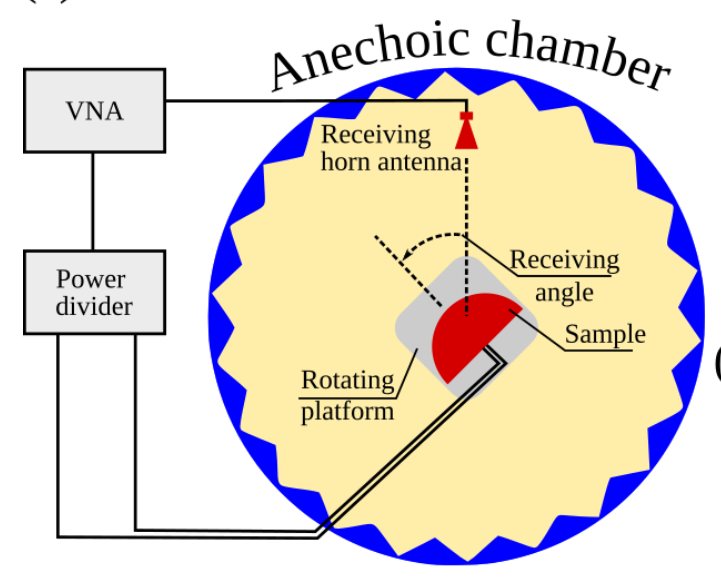

(b) ${ }^{15}-160-120-80-40 \quad 0 \quad 40 \quad 80 \quad 120 \quad 160$

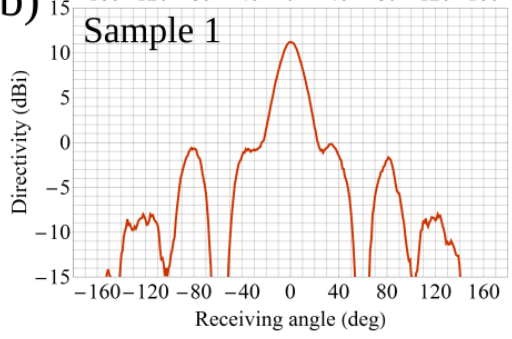

(d)

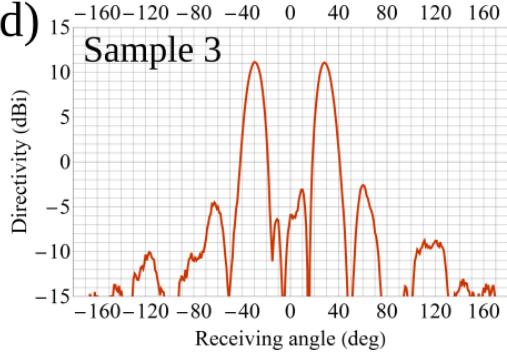

(C) 15 -160-120-80 -40 $0 \quad 40 \quad 80 \quad 120 \quad 160$
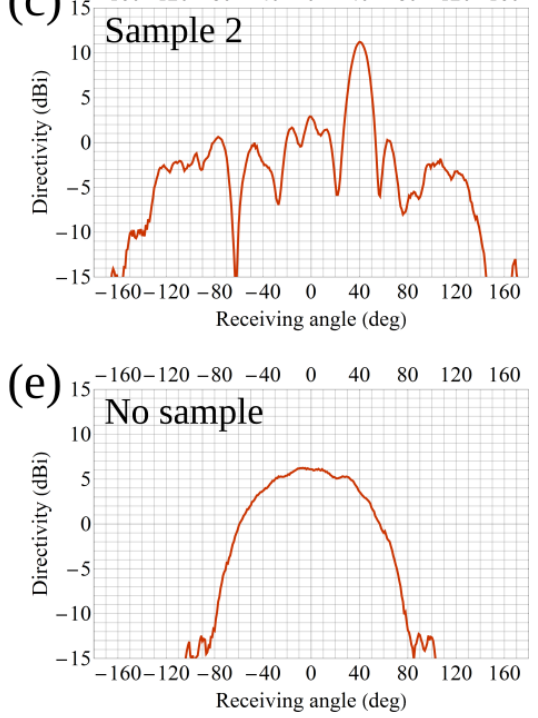

FIG. 7. (a) Schematic illustration of the experimental setup used to measure the far-field patterns. (b)-(d) Measured far-field radiation patterns set up by the three fabricated samples. (e) Measured far-field radiation pattern created by the source. Operating frequency is $5 \mathrm{GHz}$.
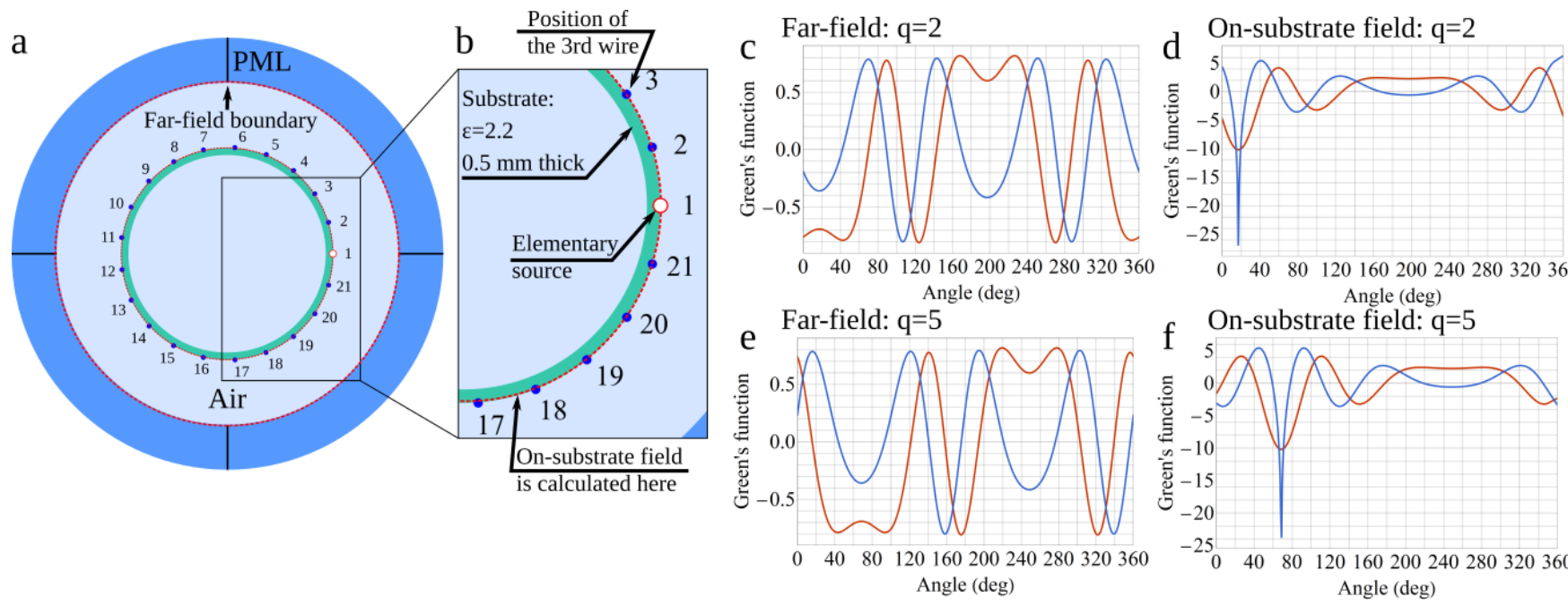

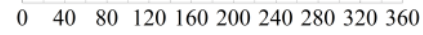
Angle (deg)

f On-substrate field: $q=5$

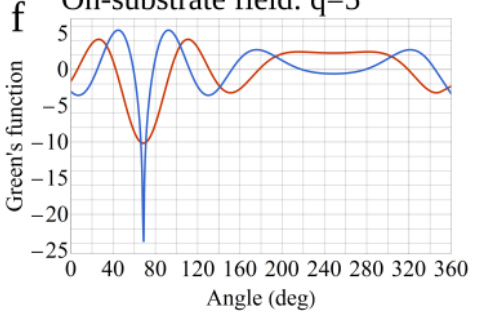

FIG. 8. (a),(b) Two-dimensional COMSOL simulation model: a cylindrical substrate of 50 mm radius is placed in the air region surrounded by a PML layer. The model is excited by an elementary source consequently placed at the positions of the wires. The elementary source is represented by a hollow disk of radius $r_{0}=0.25 / 4 \mathrm{~mm}$ (its interior is excluded from the model) with applied at the red circle electric current density boundary condition $1 /\left(2 \pi r_{0}\right) \mathrm{A} / \mathrm{m}$. (c)-(f) Greens function calculated in the far-field region (c),(e) and on the outer face of the substrate (d),(f) regions when the elementary source is at the position of the $2^{\text {nd }}(\mathrm{c}),(\mathrm{d})$ and the $5^{\text {th }}(\mathrm{e}),(\mathrm{f})$ wires.

of the source alone is shown in Fig. 7(e) to highlight the beam-forming capabilities of the designed metasurfaces.

\section{CONCLUDING REMARKS}

It is important to note that in the demonstrated numerical and experimental examples, the sparse metasurfaces are transmitting while possessing only electric response. On the other hand, conventional phase gradient approach to design transmitting metasurfaces demands implementing additionally to the electric response an effective magnetic one. It is necessary to suppress reflection and achieve $2 \pi$-range phase response to be able to establish a required phase gradient along the metasurface $\overline{13 / 7 / 8111}$. A more rigorous approach is based on engineering of electric and magnetic surface impedances 210|16|24 (and sometimes electromagnetic coupling 25|34|35) to manipulate wavefronts according to the equivalence theorem ${ }^{2 \mid 36}$. Following the 
theory presented in this study, realizing only electric response can be sufficient for an efficient control of wavefronts that might significantly simplify the design and fabrication of wavefront manipulation devices. Furthermore, intrinsic strongly non-local response of sparse metasurfaces overcomes the fundamental efficiency constraint of conventional metasurfaces imposed by the conservation of normal power flow density $17|18| 20 \mid 23$.

To conclude, we have presented a theoretical approach that opens the way to consequently design conformal sparse metasurfaces without appealing to a complex theory. Due to the versatility of the approach, one can consider different metasurface geometries and arbitrary excitation sources within the same framework. The theoretical analysis represented by Eqs. (7) and (8) allows one to approach problems of superdirectivity 37 and subdiffraction focusing 38 . Although the experiments have been performed at microwave frequencies, the theory is valid in any frequency range and may inspire research on novel applications of conformal metasurfaces. Particularly, sparse metasurfaces implemented on flexible substrates can be advantageous for realizing a reconfigurability mechanism based on mechanical deformations ${ }^{6}$. It can represent a fruitful approach to create an adaptive response without complicating a design with tunable elements (which also often bring additional ohmic losses) and bias networks.

\section{ACKNOWLEDGMENT}

The authors would like to thank Dr. Badreddine Ratni (Univ Paris Nanterre) in preparing the experimental measurement setups.

\section{Appendix A: Numerical calculation of a Green's function}

After establishing the geometry of a sparse metasurface (flat, cylindrical, or any other shape), excitation type and positions of $N$ wires, one calculates a Green's function $G_{x x}\left(r, \varphi ; \mathbf{r}_{q}\right)$ and the background field $E_{x}^{(e x t)}(r, \varphi)$ radiated by external sources. In order to find $G_{x x}\left(\mathbf{r}, \mathbf{r}_{q}\right)$, we build a $2 \mathrm{D}$ simulation model using the commercially available finite-element-method software COMSOL Multiphysics in this work. An elementary unit source is consequently placed at the different positions of loaded wires $\mathbf{r}_{q}, q=1,2, \ldots, N$. More precisely, this source is a hollow disk of radius $r_{\text {eff }}$ with an electric surface current density used as a boundary condition and set equal to $1 /\left(2 \pi r_{e f f}\right) \mathrm{A} / \mathrm{m}$. Figures 8 (a) and (b) demonstrate a schematics of a simulation model used to calculate the Greens function for the example of the cylindrical spare metasurface discussed in Section III The electric field created by the source is recorded at each position along the substrate. There is no need to know the Green's function over the whole $2 \mathrm{D}$ plane but only at certain points.

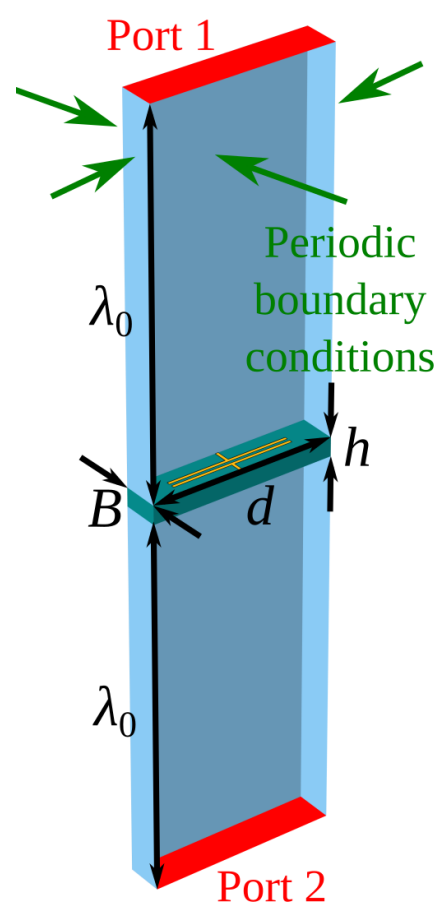

FIG. 9. Principal geometry of a 3D full-wave simulation model which is used to calculate the reflection coefficient $S_{11}$ from an array of structured wires.

Namely, we extract the electric field at the positions of the wires to construct the matrix of mutual-impedance densities $Z_{q p}^{(m)}$ and to control azimuthal wavefront at the distance $r$. In beam-forming applications, far-field calculations should be performed and the electric field in the far-field as a function of the azimuthal angle is recorded. In Figs. $8(\mathrm{c})-(\mathrm{f}), G_{x x}\left(\mathbf{r}, \mathbf{r}_{q}\right)$ for the positions of the $2^{\text {nd }}$ and $5^{\text {th }}$ wires are shown.

\section{Appendix B: Local periodic approximation}

This Section provides details on the local periodic approximation developed in Ref. ${ }^{29}$ for planar sparse metasurfaces in the reflection configuration. According to the LPA, in order to find the load-impedance density, a structured wire from a nonuniform array is placed in the corresponding uniform array of period $d$ illuminated by a plane wave incident at angle $\theta$. It allows one to retrieve the load-impedance density $Z_{q}$ from the complex amplitude of the specularly reflected TE-polarized plane wave. Indeed, a polarization current $I$ excited in a wire by the incident plane wave is linked to the complex amplitude $S_{11}$ of the specularly reflected wave via the following formula 29

$$
I=-\frac{2 d}{k \eta} \frac{\left(S_{11} e^{2 i \beta_{0}\left(h+\lambda_{0}\right)}-R_{0}^{\mathrm{TE}} e^{2 i \beta_{0} h}\right) \beta_{0}}{\left(1+R_{0}^{\mathrm{TE}}\right) e^{j \beta_{0} h}}
$$




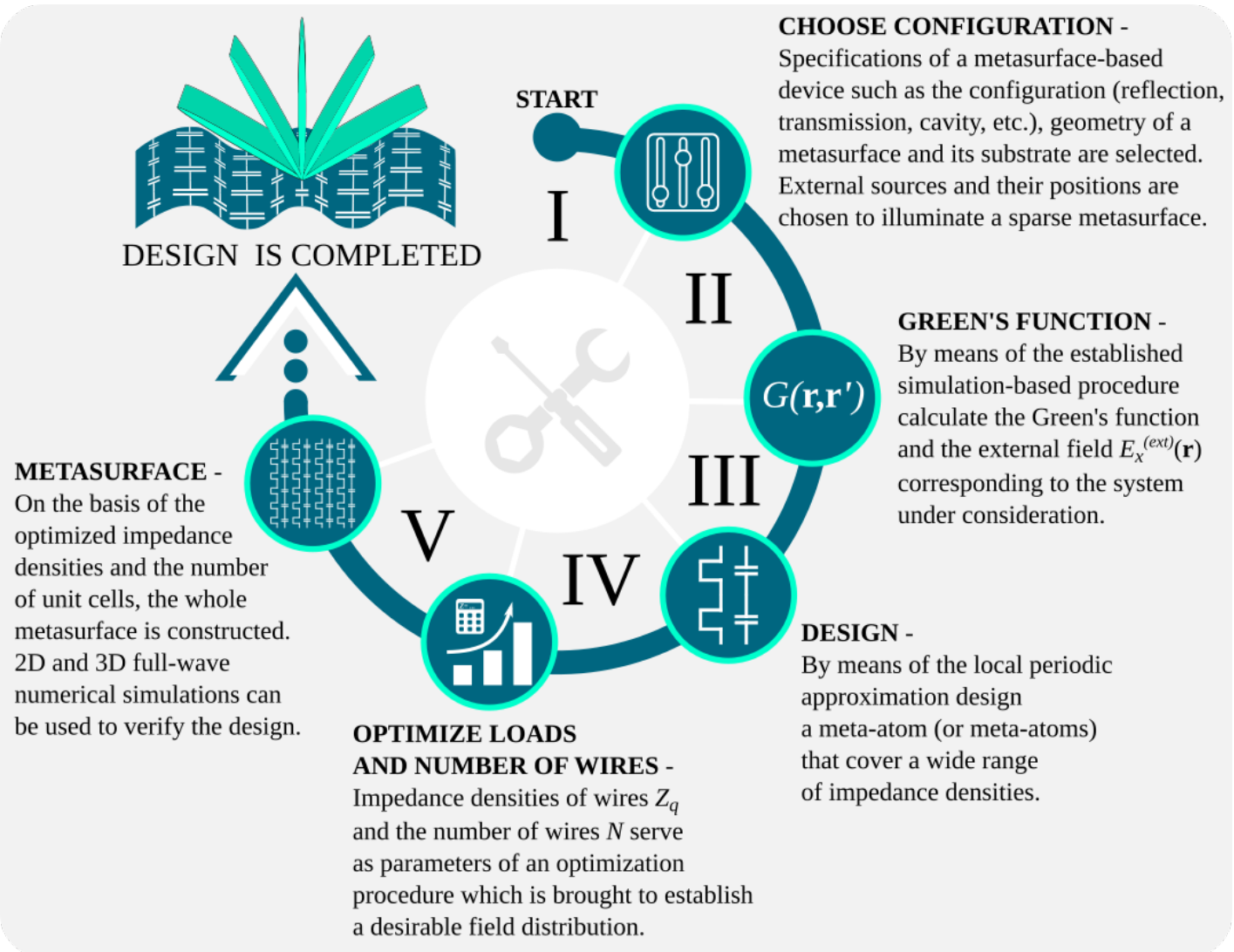

FIG. 10. A flowchart of the optimization-aided design procedure of a sparse metasurface.

where $h$ is the thickness of the substrate, $R_{m}^{\mathrm{TE}}$ is the Fresnel's reflection coefficient from the substrate

$$
R_{m}^{\mathrm{TE}}=\frac{\frac{j}{2}\left(\frac{\beta_{m}}{\beta_{m}^{s}}-\frac{\beta_{m}^{s}}{\beta_{m}}\right) \tan \left(\beta_{m}^{s} h\right)}{1+\frac{j}{2}\left(\frac{\beta_{m}}{\beta_{m}^{s}}+\frac{\beta_{m}^{s}}{\beta_{m}}\right) \tan \left(\beta_{m}^{s} h\right)},
$$

$\beta_{m}=\sqrt{k_{0}^{2}-\xi_{m}^{2}}$ and $\beta_{m}^{s}=\sqrt{\varepsilon_{s} k_{0}^{2}-\xi_{m}^{2}}$ are, respectively, normal components of the wavevector of the $m^{\text {th }}$ Floquet mode outside and inside the substrate, $\xi_{m}=k_{0} \sin (\theta)+$ $2 \pi m / d$ is the tangential component of the wavevector.

The complex amplitude of the specularly reflected wave can be obtained from $3 \mathrm{D}$ full-wave simulations. The principal geometry of a corresponding simulation model is sketched in Fig. 9 and consists of two parts: a structured wire, represented by a printed capacitor on top of a dielectric substrate, and an air region. Periodic boundary conditions are imposed on the side faces of the model. The latter is excited by a periodic Port 1 assigned to the upper face of the air region, which is highlighted with the red color in Fig. 9. The periodic Port 1 creates a plane wave incident at angle $\theta$ and is also used as a listening port to calculate the scattering parameter $S_{11}$. Periodic Port 2 is set to the listening mode to accept the transmitted wave. The thickness of the air region above and below the substrate is set to the operating vacuum wavelength $\lambda_{0}$ to eliminate higher order evanescent Floquet modes.
By modeling the polarization current excited in a structured wire as Dirac delta function $\delta(y, z)$, the interaction with the substrate and between adjacent wires can be taken into consideration analytically via the mutualimpedance density $Z_{m}{ }^{29}$

$$
Z_{m}=\frac{k \eta}{2} \sum_{n=1}^{+\infty} \cos [k \sin [\theta] n L] H_{0}^{(2)}[k n d]+\frac{k \eta}{2 d} \sum_{m=-\infty}^{+\infty} \frac{R_{m}^{T E}}{\beta_{m}}
$$

It allows one to retrieve the load-impedance density $Z_{q}$ of a wire by subtracting the electric field induced by adjacent wires and the substrate

$$
Z_{q}=\frac{E_{0}}{I}-\frac{k_{0} \eta}{4} H_{0}^{(2)}\left(k_{0} r_{e f f}\right)-Z_{m}
$$

where $E_{0}=\left(1+R_{0}^{T E}\right) \exp \left[j \beta_{0} h\right]$ represents the external electric field at the location of the central wire and induced by the incident wave and its reflection from the substrate. It should be noted that the term corresponding to $Z_{q q}^{(m)}$ in Ohm's law, Eq. (4), accounts for both the self-interaction and the interaction with a substrate and an environment, while in the LPA the mutualimpedance density subtracted on the right-hand side of Eq. (B4) corresponds only to the interaction with a substrate and other wires in the uniform array. Therefore, Eq. (B4) is accordingly modified by subtracting from 


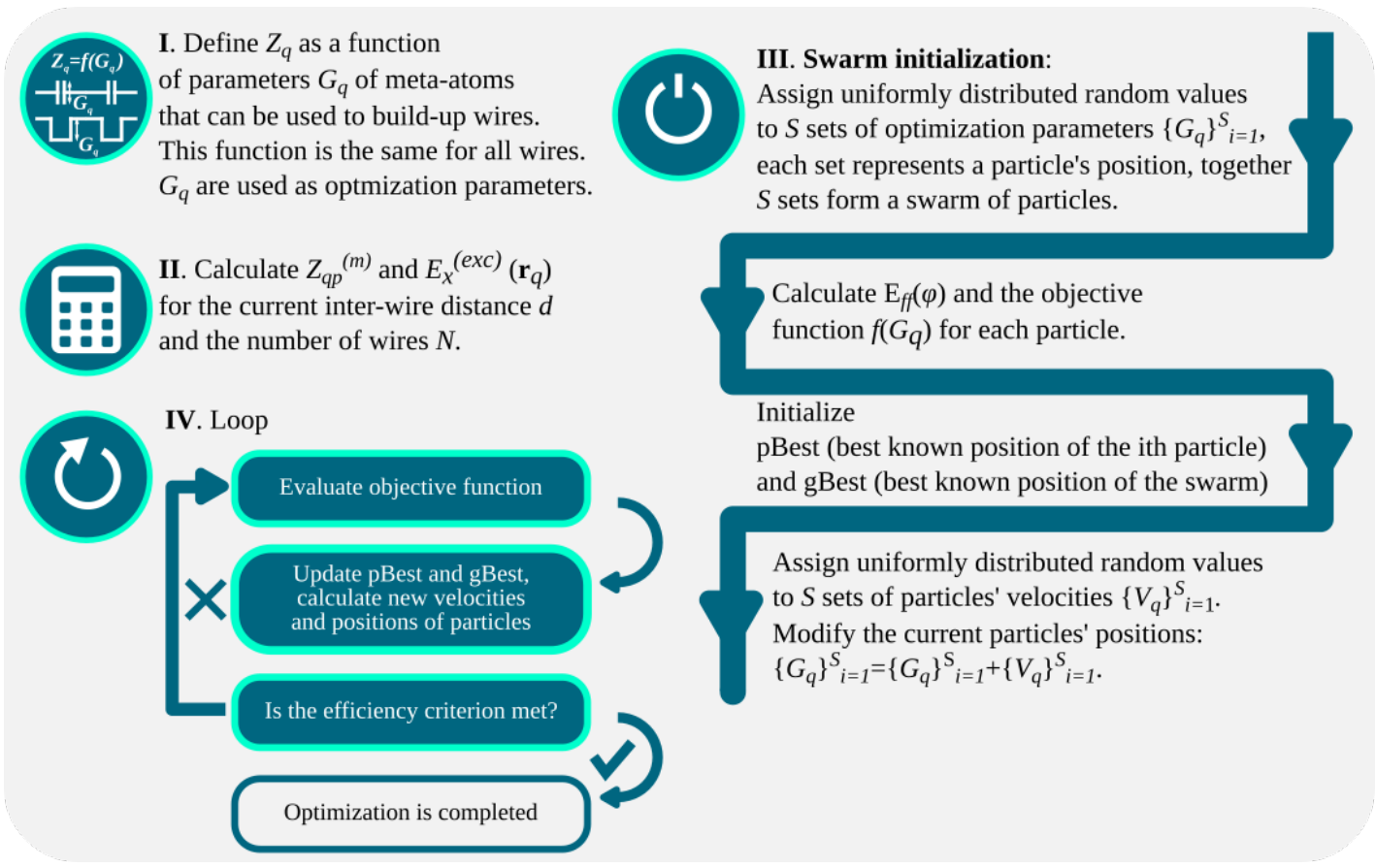

FIG. 11. A block diagram of a particle swarm optimization algorithm employed in this work to achieve a desirable radiation pattern.

the right-hand side the self-interaction term which, luckily, can be accounted by a simple analytical expression $-k_{0} \eta H_{0}^{(2)}\left(k_{0} r_{e f f}\right) / 4$.

\section{Appendix C: Optimization-aided design procedure}

This Section provides a step-by-step guide to design sparse metasurfaces by means of the LPA and an optimization procedure. The design procedure can be decomposed in five main steps, as outlined in the flowchart depicted in Fig. 10. The design procedure begins by specifying the configuration of the metasurface-based design (reflection, transmission, cavity-excited, etc.), geometry of the metasurface and its substrate. Finally, external sources and their positions are selected to efficiently illuminate the metasurface. Once the basic parameters are fixed, the second step is to calculate the Green's function and the external field by means of the established simulation-based procedure detailed in Appendix A. Then, the total electric field created by a sparse metasurface is given by Eq. (3).

The third step is independent from the previous two and requires to design a meta-atom (or meta-atoms) that will form the wires of a sparse metasurface. To that end, the local periodic approximation is used. A metaatom's design should provide a wide range of achievable impedance densities of a corresponding wire when changing its geometrical parameters (or an external bias if there are embedded tunable elements).

Each desired configuration of the diffraction pattern requires different set of impedance densities which obey Ohm's law represented by Eq. (4). In the fourth step of the procedure, one finds load-impedance densities of wires composing a sparse metasurface and required to establish a desired distribution of the electric field. In general case, an arbitrary electric field $E_{x}(\mathbf{r})$, as it is stressed in Section II] requires impedance densities that have $\operatorname{Re}\left[Z_{q}\right] \neq 0$ and imply engineering of active and/or lossy elements. Including in the period additional wires and satisfying the power conservation conditions

$$
\operatorname{Re}\left[\left(E_{x}^{(e x c)}\left(\mathbf{r}_{q}\right)-\sum_{p=1}^{N} Z_{q p}^{(m)} I_{p}\right) I_{q}^{*}\right]=\frac{k \eta}{4}\left|I_{q}\right|^{2}
$$

makes it sufficient to use purely reactive loads. On the other hand, any realistic passive meta-atom possesses inevitable resistive response whether because of conduction and/or dielectric losses or embedded lossy tunable elements (such as varactor diodes, for instance). Although the LPA allows one to account for these practical aspects of a meta-atom's design, the capacity to judiciously engineer $\operatorname{Re}\left[Z_{q}\right]$ independently from $\operatorname{Im}\left[Z_{q}\right]$ can be very limited. Furthermore, the set of equations (C1) should be modified to the following set of inequalities

$$
\operatorname{Re}\left[\left(E_{x}^{(e x c)}\left(\mathbf{r}_{q}\right)-\sum_{p=1}^{N} Z_{q p}^{(m)} I_{p}\right) I_{q}^{*}\right]>\frac{k \eta}{4}\left|I_{q}\right|^{2}
$$

which accounts for the resistance of wires and indicates that the power received by a wire is greater than the power it radiates. In many situations, it is preferable 
to minimize the impact of $\operatorname{Re}\left[Z_{q}\right]$ resulting in increased ohmic losses. Instead of elaborating on the analytical procedure developed in Section II and justifying the number of wires, the inverse scattering problem can be efficiently solved by means of a numerical optimization procedure. It can be particularly useful for beam-forming applications representing a subset of all possible far-field configurations when the power is maximized only in certain directions. Therefore, it might not be required to control all $2 M+1$ of the Fourier harmonics and a lesser number of wires than $N=2 M+1$ can be used for beamforming. As optimization parameters, the optimization procedure uses geometrical parameters of meta-atoms (or external biases) and the total number of wires. The geometrical parameters of meta-atoms $G_{q}$ are related to the load-impedance density $Z_{q}$ of a wire by means of the LPA. In their turn, load-impedance densities determine polarization currents and consequently the total electric field found from Eqs. (4) and (3). The objective function may vary depending on the needs. The one that has been used in this work to equally split the radiated power between an arbitrary number of beams when minimizing the level of secondary lobes is

$$
f\left(G_{q}\right)=t^{t}\left(\prod_{n \in \mathbb{D}}\left|E_{f f}\left(\varphi_{n}\right)\right|^{2}\right) /\left(\int_{0}^{2 \pi}\left|E_{f f}(\varphi)\right|^{2} \mathrm{~d} \varphi\right)^{t} .
$$

Here, $\mathbb{D}$ represents a discrete set of desired beams at angles $\varphi_{n}$ and $t$ is the size of this set. As a concrete optimization algorithm, a particle swarm optimization has been employed in this work, which showed better performances than a real-valued genetic algorithm. A block diagram is displayed in Fig. 11. Once the optimal loadimpedance densities are found, the fourth step of the design procedure is completed.

The fifth and the final step of the design procedure is to compose the whole metasurface out of designed metaatoms with optimized geometrical parameters and validate the final design using 3D full-wave numerical simulations. It is important to note that the developed optimization procedure itself does not include full-wave simulations and utilizes only numerical arrays calculated beforehand. It makes the optimization and the development of a final design much faster than in the case when only direct optimization of a whole metasurface is performed.

\section{Appendix D: Geometrical parameters of experimental samples}

Table I provides geometrical parameters of elements constituting the fabricated experimental samples.
* sburokur@parisnanterre.fr

1 N. Yu, P. Genevet, M. A. Kats, F. Aieta, J.-P. Tetienne, F. Capasso, and Z. Gaburro, Light propagation with phase discontinuities: Generalized laws of reflection and refraction, Science 334, 333 (2011)

2 C. Pfeiffer and A. Grbic, Metamaterial huygens' surfaces: Tailoring wave fronts with reflectionless sheets, Phys. Rev. Lett. 110, 197401 (2013)

3 D. Germain, D. Seetharamdoo, S. N. Burokur, and A. de Lustrac, Phase-compensated metasurface for a conformal microwave antenna, Applied Physics Letters 103, $124102(2013)$

${ }^{4}$ D. J. Gregoire, 3-D conformal metasurfaces, IEEE Antennas and Wireless Propagation Letters 12, 233 (2013)

L. Liang, M. Qi, J. Yang, X. Shen, J. Zhai, W. Xu, B. Jin, W. Liu, Y. Feng, C. Zhang, H. Lu, H.-T. Chen, L. Kang, W. Xu, J. Chen, T. J. Cui, P. Wu, and S. Liu, Anomalous terahertz reflection and scattering by flexible and conformal coding metamaterials, Advanced Optical Materials 3, $1374(2015)$

o S. Walia, C. M. Shah, P. Gutruf, H. Nili, D. R. Chowdhury, W. Withayachumnankul, M. Bhaskaran, and S. Sriram, Flexible metasurfaces and metamaterials: A review of materials and fabrication processes at micro- and nanoscales, Applied Physics Reviews 2, 011303 (2015).

7 J. Cheng, S. Jafar-Zanjani, and H. Mosallaei, All-dielectric ultrathin conformal metasurfaces: lensing and cloaking applications at $532 \mathrm{~nm}$ wavelength, Scientific reports 6, 38440 (2016).
8 S. M. Kamali, A. Arbabi, E. Arbabi, Y. Horie, and A. Faraon, Decoupling optical function and geometrical form using conformal flexible dielectric metasurfaces, Nature communications 7, 11618 (2016).

9 H.-X. Xu, S. Tang, C. Sun, L. Li, H. Liu, X. Yang, F. Yuan, and Y. Sun, High-efficiency broadband polarizationindependent superscatterer using conformal metasurfaces, Photon. Res. 6, 782 (2018)

$10 \mathrm{~K}$. Wu, P. Coquet, Q. J. Wang, and P. Genevet, Modelling of free-form conformal metasurfaces, Nature communications 9, 3494 (2018).

11 H. Li, C. Ma, F. Shen, K. Xu, D. Ye, J. Huangfu, C. Li, L. Ran, and T. A. Denidni, Wide-angle beam steering based on an active conformal metasurface lens, IEEE Access 7, 185264 (2019)

${ }_{12}$ P. Nayeri, F. Yang, and A. Z. Elsherbeni, Reflectarray Antennas: Theory, Designs and Applications (Wiley Online Library, 2018).

13 V. S. Asadchy, M. Albooyeh, S. N. Tcvetkova, A. DíazRubio, Y. Ra'di, and S. A. Tretyakov, Perfect control of reflection and refraction using spatially dispersive metasurfaces, Phys. Rev. B 94, 075142 (2016).

14 N. Mohammadi Estakhri and A. Alù, Wave-front transformation with gradient metasurfaces, Phys. Rev. X 6, 041008 (2016)

15 E. F. Kuester, M. A. Mohamed, M. Piket-May, and C. L. Holloway, Averaged transition conditions for electromagnetic fields at a metafilm, IEEE Transactions on Antennas and Propagation 51, 2641 (2003) 
16 A. Epstein and G. V. Eleftheriades, Huygens' metasurfaces via the equivalence principle: design and applications, J. Opt. Soc. Am. B 33, A31 (2016)

17 Y. Ra'di, D. L. Sounas, and A. Alù, Metagratings: Beyond the limits of graded metasurfaces for wave front control, Phys. Rev. Lett. 119, 067404 (2017)

18 A. Epstein and O. Rabinovich, Unveiling the properties of metagratings via a detailed analytical model for synthesis and analysis, Phys. Rev. Applied 8, 054037 (2017)

19 A. M. H. Wong and G. V. Eleftheriades, Perfect anomalous reflection with a bipartite huygens' metasurface, Phys. Rev. X 8, 011036 (2018).

${ }^{20}$ V. Popov, F. Boust, and S. N. Burokur, Constructing the near field and far field with reactive metagratings: Study on the degrees of freedom, Phys. Rev. Applied 11, 024074 (2019)

21 A. Epstein and G. V. Eleftheriades, Synthesis of passive lossless metasurfaces using auxiliary fields for reflectionless beam splitting and perfect reflection, Phys. Rev. Lett. 117, 256103 (2016)

22 A. Díaz-Rubio, V. S. Asadchy, A. Elsakka, and S. A. Tretyakov, From the generalized reflection law to the realization of perfect anomalous reflectors, Science Advances 3, 10.1126/sciadv.1602714 (2017).

23 D. Kwon, Lossless scalar metasurfaces for anomalous reflection based on efficient surface field optimization, IEEE Antennas and Wireless Propagation Letters 17, 1149 (2018)

${ }_{24}$ A. Epstein and G. V. Eleftheriades, Passive lossless huygens metasurfaces for conversion of arbitrary source field to directive radiation, IEEE Transactions on Antennas and Propagation 62, 5680 (2014)

${ }^{25}$ A. Epstein and G. V. Eleftheriades, Arbitrary powerconserving field transformations with passive lossless omega-type bianisotropic metasurfaces, IEEE Transactions on Antennas and Propagation 64, 3880 (2016)

26 Indeed, the matrix composed of coefficients $C_{n}^{(q)}$ is not degenerate since $G_{x x}\left(r, \phi ; \mathbf{r}_{q}\right)$ related to different points $\mathbf{r}_{q}$ are linearly independent. On the other hand symmetries of the systems should be respected, e.g. a linear array of wires in vacuum radiates symmetrically and there is an additional constraint between the Fourier coefficients
$C_{n}=C_{-n}$.

27 X. Wang, A. Díaz-Rubio, A. Sneck, A. Alastalo, T. Mäkelä, J. Ala-Laurinaho, J. Zheng, A. V. Räisänen, and S. A. Tretyakov, Systematic design of printable metasurfaces: Validation through reverse-offset printed millimeter-wave absorbers, IEEE Transactions on Antennas and Propagation 66, 1340 (2018)

28 S. Tretyakov, Analytical Modeling in Applied Electromagnetics (Artech House, Norwood, MA, 2003).

29 V. Popov, M. Yakovleva, F. Boust, J.-L. Pelouard, F. Pardo, and S. N. Burokur, Designing metagratings via local periodic approximation: From microwaves to infrared, Phys. Rev. Applied 11, 044054 (2019)

30 V. Popov, F. Boust, and S. N. Burokur, Beamforming with metagratings at microwave frequencies: Design procedure and experimental demonstration, IEEE Transactions on Antennas and Propagation 68, 1533 (2020).

${ }^{31}$ P. Katehi and N. Alexopoulos, On the modeling of electromagnetically coupled microstrip antennas-the printed strip dipole, IEEE Transactions on Antennas and Propagation 32, 1179 (1984)

32 A. Epstein, J. P. S. Wong, and G. V. Eleftheriades, Cavityexcited huygens metasurface antennas for near-unity aperture illumination efficiency from arbitrarily large apertures, Nature communications 7, 10360 (2016).

33 J. Kennedy and R. Eberhart, Particle swarm optimization, in Proceedings of ICNN'95 - International Conference on Neural Networks, Vol. 4 (1995) pp. 1942-1948 vol.4.

34 V. S. Asadchy, Y. Ra'di, J. Vehmas, and S. A. Tretyakov, Functional metamirrors using bianisotropic elements, Phys. Rev. Lett. 114, 095503 (2015).

35 V. S. Asadchy, A. Díaz-Rubio, and S. A. Tretyakov, Bianisotropic metasurfaces: physics and applications, Nanophotonics 7, 1069 (2018).

36 S. A. Schelkunoff, Some equivalence theorems of electromagnetics and their application to radiation problems, The Bell System Technical Journal 15, 92 (1936)

37 S. A. Schelkunoff, A mathematical theory of linear arrays, The Bell System Technical Journal 22, 80 (1943)

38 K. Huang, F. Qin, H. Liu, H. Ye, C.-W. Qiu, M. Hong, B. Luk'yanchuk, and J. Teng, Planar diffractive lenses: Fundamentals, functionalities, and applications, Advanced Materials 30, 1704556 (2018) 
TABLE I. Geometrical parameters of the samples. Schematics of capacitor and inductor elements is illustrated in Fig. 6(a). The parameter $w$ is the same for all elements and equals to $0.25 \mathrm{~mm}$, the thickness of the copper cladding is $35 \mu \mathrm{m}$.

\begin{tabular}{|c|c|c|c|c|c|c|c|c|c|}
\hline \multirow{2}{*}{ Element number } & \multicolumn{3}{|c|}{ Sample 1} & \multicolumn{3}{|c|}{ Sample 2} & \multicolumn{3}{|c|}{ Sample 3} \\
\hline & Element type & $\mathrm{B}(\mathrm{mm})$ & $\mathrm{A}(\mathrm{mm})$ & Element type & $\mathrm{B}(\mathrm{mm})$ & $\mathrm{A}(\mathrm{mm})$ & Element type & B $(\mathrm{mm})$ & $\mathrm{A}(\mathrm{mm})$ \\
\hline 1 & capacitor & 10 & 3,31 & capacitor & 10 & 2,58 & capacitor & 10 & 1,39 \\
\hline 2 & capacitor & 10 & 4,78 & capacitor & 10 & 5,68 & capacitor & 5 & 1,04 \\
\hline 3 & capacitor & 10 & 1,58 & capacitor & 10 & 4,46 & capacitor & 10 & 3,47 \\
\hline 4 & inductor & 10 & 5,45 & capacitor & 5 & 1,06 & capacitor & 5 & 1,30 \\
\hline 5 & capacitor & 10 & 4,81 & capacitor & 5 & 1,86 & capacitor & 10 & 0,53 \\
\hline 6 & capacitor & 5 & 0,88 & capacitor & 10 & 4,46 & capacitor & 10 & 4,72 \\
\hline 7 & capacitor & 5 & 1,99 & capacitor & 10 & 2,32 & inductor & 5 & 7,29 \\
\hline 8 & capacitor & 10 & 4,73 & capacitor & 10 & 4,73 & capacitor & 5 & 1,53 \\
\hline 9 & capacitor & 10 & 2,41 & inductor & 10 & 5,62 & capacitor & 5 & 1,18 \\
\hline 10 & capacitor & 10 & 1,16 & capacitor & 5 & 0,45 & capacitor & 10 & 0,56 \\
\hline 11 & capacitor & 10 & 4,31 & capacitor & 10 & 7,64 & capacitor & 10 & 1,33 \\
\hline 12 & capacitor & 10 & 3,30 & capacitor & 10 & 3,85 & capacitor & 5 & 1,07 \\
\hline 13 & capacitor & 10 & 0,60 & capacitor & 5 & 1,29 & capacitor & 5 & 1,04 \\
\hline 14 & capacitor & 10 & 3,52 & capacitor & 10 & 2,78 & capacitor & 10 & 6,89 \\
\hline 15 & capacitor & 10 & 3,42 & capacitor & 10 & 4,72 & capacitor & 5 & 0,97 \\
\hline 16 & capacitor & 10 & 3,52 & capacitor & 10 & 1,64 & capacitor & 10 & 6,89 \\
\hline 17 & capacitor & 10 & 0,60 & inductor & 10 & 4,20 & capacitor & 5 & 1,04 \\
\hline 18 & capacitor & 10 & 3,30 & capacitor & 10 & 0,84 & capacitor & 5 & 1,07 \\
\hline 19 & capacitor & 10 & 4,31 & capacitor & 10 & 0,74 & capacitor & 10 & 1,33 \\
\hline 20 & capacitor & 10 & 1,16 & capacitor & 10 & 3,57 & capacitor & 10 & 0,56 \\
\hline 21 & capacitor & 10 & 2,41 & capacitor & 5 & 0,82 & capacitor & 5 & 1,18 \\
\hline 22 & capacitor & 10 & 4,73 & inductor & 10 & 3,00 & capacitor & 5 & 1,53 \\
\hline 23 & capacitor & 5 & 1,99 & capacitor & 10 & 2,25 & inductor & 5 & 7,29 \\
\hline 24 & capacitor & 5 & 0,88 & capacitor & 10 & 3,34 & capacitor & 10 & 4,72 \\
\hline 25 & capacitor & 10 & 4,81 & capacitor & 5 & 1,29 & capacitor & 10 & 0,53 \\
\hline 26 & inductor & 10 & 5,45 & capacitor & 10 & 1,41 & capacitor & 5 & 1,30 \\
\hline 27 & capacitor & 10 & 1,58 & capacitor & 10 & 0,65 & capacitor & 10 & 3,47 \\
\hline 28 & capacitor & 10 & 4,78 & capacitor & 10 & 0,35 & capacitor & 5 & 1,04 \\
\hline 29 & capacitor & 10 & 3,31 & capacitor & 5 & 1,49 & capacitor & 10 & 1,39 \\
\hline
\end{tabular}

